\title{
IMPROVING AUDITORY ATTENTION DECODING PERFORMANCE OF LINEAR AND NON-LINEAR METHODS USING STATE-SPACE MODEL
}

\author{
Ali Aroudi Tobias de Taillez $\quad$ Simon Doclo \\ Department of Medical Physics and Acoustics and Cluster of Excellence Hearing4All, \\ University of Oldenburg, Oldenburg, Germany \\ ali.aroudi@uni-oldenburg.de
}

\begin{abstract}
Identifying the target speaker in hearing aid applications is crucial to improve speech understanding. Recent advances in electroencephalography (EEG) have shown that it is possible to identify the target speaker from single-trial EEG recordings using auditory attention decoding (AAD) methods. AAD methods reconstruct the attended speech envelope from EEG recordings, based on a linear least-squares cost function or non-linear neural networks, and then directly compare the reconstructed envelope with the speech envelopes of speakers to identify the attended speaker using Pearson correlation coefficients. Since these correlation coefficients are highly fluctuating, for a reliable decoding a large correlation window is used, which causes a large processing delay. In this paper, we investigate a state-space model using correlation coefficients obtained with a small correlation window to improve the decoding performance of the linear and the non-linear AAD methods. The experimental results show that the state-space model significantly improves the decoding performance.
\end{abstract}

Index Terms - auditory attention decoding, state-space model, neural network, EEG signal, brain computer interface

\section{INTRODUCTION}

Multi-microphone speech enhancement algorithms in currently available hearing aid devices are able to perform source separation and reduce background noise to improve speech intelligibility. However, the performance of these algorithms in improving speech intelligibility typically depends on correctly identifying the target speaker to be enhanced. In hearing aid applications, the target speaker is typically identified using assumptions such as the target speaker being located in front of the listener or being the loudest speaker. However, since in real-world conditions these assumptions may often be violated, e.g., when the auditory attention of the listener is misaligned with the assumptions, the performance of speech enhancement methods decreases and results in a substantially reduced benefit from hearing aids.

Recent advances in electroencephalography (EEG) have shown that it is possible to identify the target speaker from single-trial EEG recordings of a listener by decoding the auditory attention [1-3]. Several auditory attention decoding (AAD) methods have been proposed to identify the target speaker, based on, e.g., a linear least-squares cost function [4-7] and non-linear neural networks [8. 9]. The linear least-squares-based AAD method proposed in [4] is able to exploit the linear neural process of attention along the auditory pathway to identify the attended speaker. The non-linear neural-network-based AAD method proposed in [8] is able to exploit the non-linear neural process of attention in addition to the linear

This work was supported by the Deutsche Forschungsgemeinschaft (DFG, German Research Foundation) - Project ID 390895286 EXC 2177/1. neural process. To identify the attended speaker, these methods aim at reconstructing the attended speech envelope from the EEG recordings using a trained spatio-temporal estimator. In the training step, a spatio-temporal envelope estimator is trained by either minimizing the least-squares error or maximizing the correlation cost function between the attended speech envelope and the reconstructed envelope. In the decoding step, the attended speech envelope is reconstructed using the trained envelope estimator and then directly compared with the speech envelopes of two speakers using Pearson correlation coefficients to identify the attended speaker. Since these correlation coefficients are highly fluctuating, for a reliable decoding a large correlation window on the order of 30 seconds is typically used, which causes a large processing delay and hence limits the feasibility of AAD for hearing aid applications. In [7], it has been proposed to use coefficients of least-squares-based envelope estimators, obtained separately for reconstructing the attended and the unattended speech envelope. Using coefficients of estimators, a state-space model then identifies the attended speaker. In this paper, we investigate a state-space model using correlation coefficients obtained with a small correlation window to improve the decoding performance of the (linear) least-squares-based AAD method and the (non-linear) neural-network-based AAD method. The correlation coefficients are generated using either the least-squares-based AAD method or the neural-network-based AAD method. The state-space model then translates the generated correlation coefficients into smooth estimates of the attention state, based on which the attended speaker is identified.

For an acoustic scenario with two competing speakers and diffuse noise at different SNRs and reverberation times, 64-channel EEG responses with 18 participants were recorded. The experimental results show for correlation coefficients obtained with a 5 -second correlation window that the least-squares-based AAD method and the neural-network-based AAD method yield a low decoding performance. However, when using the state-space model with the least-squares-based AAD method, the decoding performance significantly improves.

\section{AUDITORY ATTENTION DECODING}

This section presents the auditory attention decoding using a state-space model, which employs correlation coefficients generated either by the least-squares-based $\mathrm{AAD}$ method and the neural-network-based AAD method. In Section 2.1 the acoustic scenario and the notation are defined. Section 2.2 describes the state-space model. Section 2.3 and Section 2.4 describe the least-squares-based AAD method and the neural-network-based AAD method.

\subsection{Configuration and notation}

We consider an acoustic scenario comprising two competing speakers and background noise in a reverberant environment, where the ongoing EEG 


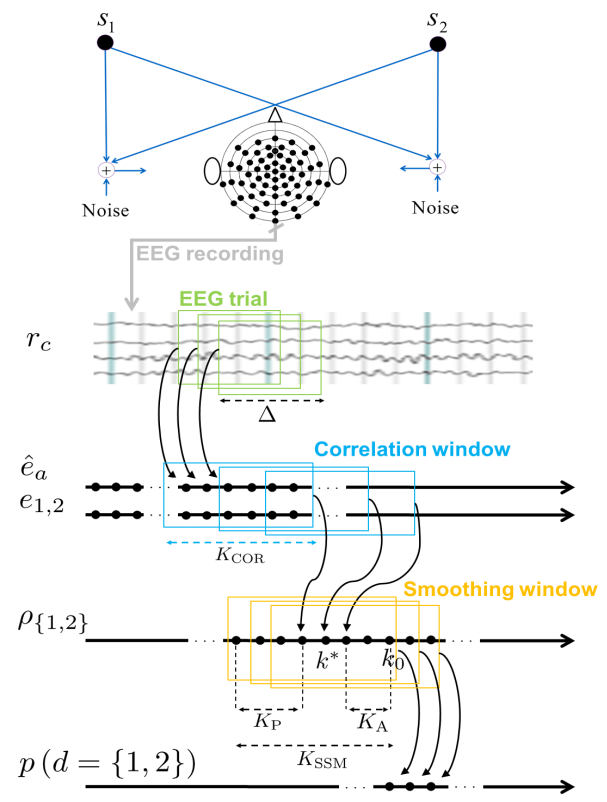

Fig. 1. Illustration of the process flow of AAD using state-space model.

responses of a listener to these acoustic stimuli are recorded (See Fig. 1). The clean speech signal of speaker 1 is denoted as $s_{1}[n]$, with $n$ the discrete time index, while the clean speech signal of speaker 2 is denoted as $s_{2}[n]$. The envelopes of the clean speech signals of speaker 1 and 2 are denoted as $e_{1}[k]$ and $e_{2}[k]$, with $k$ the sub-sampled time index, respectively.

The reconstructed attended speech envelope from $C$-channel EEG recordings $r_{c}[k]$, with $c=1 \ldots C$, using a trained spatio-temporal envelope estimator $F$ is given by

$$
\hat{e}_{a}[k]=F(\mathbf{r}[k])
$$

with

$$
\begin{gathered}
\mathbf{r}[k]=\left[\mathbf{r}_{1}^{T}[k] \mathbf{r}_{2}^{T}[k] \ldots \mathbf{r}_{C}^{T}[k]\right]^{T}, \\
\mathbf{r}_{c}[k]=\left[r_{c}[k] r_{c}[k+1] \ldots r_{c}[k+\Delta]\right]^{T},
\end{gathered}
$$

where $\Delta$ denotes the latency considered for modeling the attentional effect in the EEG responses to acoustic stimuli.

The Pearson correlation coefficients between the reconstructed attended envelope $\hat{e}_{a}[k]$ and the envelope of two speakers are given by

$$
\rho_{1, k}=\rho\left(\mathbf{e}_{1}[k], \hat{\mathbf{e}}_{a}[k]\right), \rho_{2, k}=\rho\left(\mathbf{e}_{2}[k], \hat{\mathbf{e}}_{a}[k]\right),
$$

where $\hat{\mathbf{e}}_{a}[k]$ denotes the stacked vector of the reconstructed attended envelope corresponding to a correlation window of length $K_{\mathrm{COR}}$, i.e.,

$$
\hat{\mathbf{e}}_{a}[k]=\left[\hat{e}_{a}\left[(k-1) K_{\mathrm{COR}}+1\right] \hat{e}_{a}\left[(k-1) K_{\mathrm{COR}}+2\right] \ldots \hat{e}_{a}\left[k K_{\mathrm{COR}}\right]\right]^{T},
$$

and $\mathbf{e}_{1}[k]$ and $\mathbf{e}_{2}[k]$ are defined similarly as in 5 . Please note that in this paper we assume that the clean speech signal of speakers are available for obtaining the envelopes of speakers $e_{1}[k]$ and $e_{2}[k]$. However, since in practice only microphone signals containing a mixture of speakers and ambient noise are available, the clean speech signal of speakers needs to be appropriately estimated from microphone signals, e.g., by using the noise reduction and source separation algorithms proposed in [2 3.10 11].

\subsection{AAD using state-space model}

Suppose the attended envelope is reconstructed using a trained (linear or nonlinear) spatio-temporal estimator and the correlation coefficients of speakers are obtained. We aim at estimating the probability of attending to speaker 1 or 2 based on a state-space model using the past and the subsequent correlation coefficients (see Fig. 1). Let the attention state of the listener when attending to either speaker 1 or 2 be defined as a binary random variable, i.e.,

$$
\left\{\begin{array}{l}
d_{k}=1, \quad \text { when attending to speaker } 1 \\
d_{k}=2, \quad \text { when attending to speaker } 2
\end{array},\right.
$$

which follows a Bernoulli process. The probability of attending to speakers based on the state-space model is obtained as [7. 12]

$$
p\left(d_{k}=1\right)=1-p\left(d_{k}=2\right)=\frac{1}{1+e^{-\left(z_{k}\right)}},
$$

with

$$
\begin{gathered}
z_{k}=c_{0} z_{k-1}+w_{k}, \\
w_{k} \sim \mathcal{N}\left(0, \eta_{k}\right), \\
\eta_{k} \sim \text { Inverse-Gamma }\left(a_{0}, b_{0}\right),
\end{gathered}
$$

, $c_{0}$ denoting the hyperparameter ensuring the stability of $z_{k}$, and $a_{0}$ and $b_{0}$ denoting the hyperparameters used to control the smoothing degree of the state-space model by tuning the variations of $z_{k}$ and $p\left(d_{k}=\{1,2\}\right)$. The autoregressive model in (8) implies that the (attention state) parameter $z_{k}$ at instance $k$ is predicted from $z_{k-1}$ at the instance $k-1$ with some uncertainty, which is modeled by the noise process $w(k)$. Please note that when $z_{k}$ varies from $-\infty$ to $\infty, p\left(d_{k}=1\right)$ monotonically varies from 0 to 1 . To relate the correlation coefficients of speakers to the attention state, the probability of the absolute values of correlation coefficients given attending to either speaker 1 or 2 is modeled using a Log-Normal distribution, i.e.,

$$
p\left(\left|\rho_{l, k}\right| \mid d_{k}=l\right) \sim \log -\operatorname{Normal}\left(\boldsymbol{\alpha}_{a}\right), \quad l=1,2
$$

with $\boldsymbol{\alpha}_{a}$ denoting the parameter set of the attended Log-Normal distribution. The probability of the correlation coefficients given ignoring either speaker 1 or 2 is modeled as

$$
p\left(\left|\rho_{l, k}\right| \mid d_{k} \neq l\right) \sim \log -\operatorname{Normal}\left(\boldsymbol{\alpha}_{u}\right), \quad l=1,2
$$

with $\boldsymbol{\alpha}_{u}$ denoting the parameter set of the unattended Log-Normal distribution.

Let's suppose we are at the instance $k=k_{0}$ (see Fig. 11 and aim to estimate the probability of attending to speakers $p\left(d_{k}=\{1,2\}\right)$ at the instance $k=k^{*}$ using the correlation coefficients obtained within a sliding smoothing window of length $K_{\mathrm{SSM}}=K_{P}+K_{A}+1$, with $K_{P}$ and $K_{A}$ denoting the parameters determining the number of the correlation coefficient prior to and after the instance $k^{*}$, respectively. The parameters of the state-space model corresponding to the smoothing win-

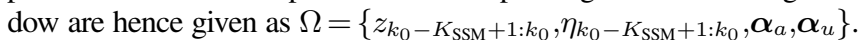
Theses parameters including $z_{k^{*}}$ are estimated from the correlation

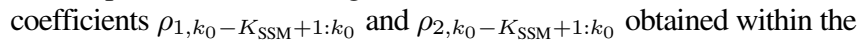
smoothing window using the Expectation Maximization (EM) estimation algorithm proposed in [7 12]. Based on the estimated attention state parameter $z_{k^{*}}$, the probability of attending to speakers $p\left(d_{k^{*}}=\{1,2\}\right)$ are obtained. It is then decided that the listener attended to speaker 1 if $p\left(d_{k^{*}}=1\right)>p\left(d_{k^{*}}=2\right)$ or attended to speaker 2 otherwise. Please note that the estimated parameters $\Omega$ are also used for the initialization of parameters in the next smoothing window.

In the simulations (see Section 3), we will consider to use the state-space model with correlation coefficients generated either by the least-squares-based AAD method (see Section 2.3) or the neural-networkbased AAD method (see Section 2.4). 


\subsection{Least-squares-based AAD}

The least-squares-based AAD method proposed in [4] aims at estimating the attended speech envelope from the EEG recordings using a trained linear spatio-temporal estimator. In the training step, the attended speaker is assumed to be known and an attended speech signal is used to train a linear estimator by minimizing the least-squares error between the attended speech envelope $e_{a}[k]$ and the reconstructed envelope $\hat{e}_{a}[k]$, i.e.,

$$
\min _{\mathbf{g}} \frac{1}{K} \sum_{k=1}^{K}\left(e_{a}[k]-\hat{e}_{a}[k]\right)^{2}+\beta \mathbf{g}^{T} \mathbf{D g},
$$

with $\hat{e}_{a}[k]=F(\mathbf{r}[k])=\mathbf{g}^{T} \mathbf{r}[k], \mathbf{D}$ denoting the derivative matrix [13] and $\beta$ denoting a regularization parameter. The linear estimator minimizing the regularized least-squares cost function in 13 is equal to

$$
\mathbf{g}=(\mathbf{Q}+\beta \mathbf{D})^{-1} \mathbf{q}
$$

with the correlation matrix $\mathbf{Q}$ and the cross-correlation vector $\mathbf{q}$ given by

$$
\mathbf{Q}=\frac{1}{K} \sum_{k=1}^{K}\left(\mathbf{r}[k] \mathbf{r}^{T}[k]\right), \mathbf{q}=\frac{1}{K} \sum_{k=1}^{K}\left(\mathbf{r}[k] e_{a}[k]\right) .
$$

In the decoding step, the attended envelope $\hat{e}_{a}[k]$ is obtained using the (trained) linear estimator $\mathbf{g}$ in (14). Next, the correlation coefficients between the reconstructed attended envelope and the envelope of two speaker $\rho_{1, k}$ and $\rho_{2, k}$ are computed as in (4). Based on these correlation coefficients, it is then decided that the listener attended to speaker 1 if $\rho_{1, k}>\rho_{2, k}$ or attended to speaker 2 otherwise.

\subsection{Neural-network-based AAD}

The neural-network-based AAD method aims at estimating the attended speech envelope from the EEG recordings using a trained non-linear spatio-temporal estimator. Similarly as in [8.9], we consider a network $\mathcal{H}$ consisting of a hidden convolutional layer with hyperbolic tangent activation functions and one output layer with linear activation functions. In the training step, the network is trained to maximize the correlation between the attended speech envelope and the reconstructed envelope by minimizing the correlation cost function [8], i.e.,

$$
\min \frac{1}{K} \sum_{k=1}^{K}\left(1-\rho\left(\mathbf{e}_{a}[k], \hat{\mathbf{e}}_{a}[k]\right)\right),
$$

A correlation cost function equal to 0 corresponds to the maximum correlation between the attended speech envelope and the reconstructed envelope, i.e., $\rho\left(\mathbf{e}_{a}[k], \hat{\mathbf{e}}_{a}[k]\right)=1$, while a correlation cost function equal to 1 corresponds to the minimum correlation. A correlation cost function larger than 1 corresponds to a negative correlation.

In the decoding step, the attended envelope is obtained using the (trained) network $\mathcal{H}$, i.e., $\hat{e}_{a}[k]=F(\mathbf{r}[k])=\mathcal{H}(\mathbf{r}[k])$. Next, the correlation coefficients between the reconstructed attended envelope and the envelope of two speaker are computed $\rho_{1, k}$ and $\rho_{2, k}$ as in (4). Based on these correlation coefficients, it is then decided that the listener attended to speaker 1 if $\rho_{1, k}>\rho_{2, k}$ or attended to speaker 2 otherwise.

\section{EXPERIMENTAL SETUP}

\subsection{Acoustic stimuli and EEG measurement}

EEG responses were recorded for 18 native German-speaking participants. Two German audio stories, uttered by two different male speakers, were simultaneously presented to the participants using insert earphones. The presented stimuli at both ears were generated by convolving the clean speech signals, i.e., the audio stories, with (non-individualized) binaural impulse responses from [14], and adding diffuse noise, generated according to [15]. The left and the right speaker were simulated at $\theta_{1}=-45^{\circ}$ and $\theta_{2}=45^{\circ}$. Eight different acoustic conditions were considered for the stimuli: one anechoic condition with no background noise, two reverberant conditions with a moderate and a large reverberation time (reverberation time $T_{60}=0.5 \mathrm{~s}$ and $1 \mathrm{~s}$ ), two anechoic conditions with binaural input SNRs $=$ $9.0 \mathrm{~dB}$ and $4.0 \mathrm{~dB}$, and three combinations of reverberation and noise. Among all participants, 8 participants were instructed to attend to the left speaker, while 10 participants were instructed to attend to the right speaker. Two participants were excluded from the analysis, one participant due to poor attentional performance and the other one due to a technical hardware problem. The EEG responses were recorded using $C=64$ channels at a sampling frequency of $500 \mathrm{~Hz}$, and referenced to the nose electrode. The EEG responses were re-referenced offline to a common average reference, band-pass filtered between $2 \mathrm{~Hz}$ and $8 \mathrm{~Hz}$ using a third-order Butterworth band-pass filter, and subsequently downsampled to $64 \mathrm{~Hz}$. The envelopes of the speech signals were obtained using a Hilbert transform, followed by low-pass filtering at $8 \mathrm{~Hz}$ and downsampling to $64 \mathrm{~Hz}$.

\subsection{AAD training and testing}

For AAD training and testing, the EEG recordings for the different acoustic conditions were grouped together based on acoustic similarity, resulting in four experimental analysis conditions, i.e., anechoic, reverberant, anechoic-noisy, and reverberant-noisy, each of length 20 minutes. To avoid using EEG recordings of the same experimental analysis condition for training and testing, the leave-one-condition-out approach was used, i.e., four combinations of three experimental analysis conditions without repetition were considered for training and the left condition for each combination was considered for testing. This resulted in four training conditions and four testing conditions.

For the least-squares-based AAD method, the latency parameter of the linear estimator in (3) was set to $\Delta=20$ (corresponding to $312 \mathrm{~ms}$ ), as found to be an appropriate choice for AAD [4 16]. For training, the estimator in 14 and the regularization parameter $\beta$ of the estimator in 13 was determined using a k-fold cross-validation approach with $k=10$, each of length 6 minutes. For testing, the EEG recordings were segmented into trials of length $5 \mathrm{~s}$ with an overlap of $4.98 \mathrm{~s}$ (corresponding to one sample shift). The correlation coefficients were computed using a correlation window of length $K_{\mathrm{COR}}=5 \mathrm{~s}$ with an overlap of $4.5 \mathrm{~s}$.

For the neural-network-based AAD method, the network $\mathcal{H}$ consisting of a convolutional hidden layer with a filter kernel size of 20 samples (corresponding to $312 \mathrm{~ms}$ ) was used. For training, the network was trained using a k-fold cross-validation approach with $k=10$, each of length 6 minutes. The network was trained with the Nadam optimizer [17] using a batch size of 3840 samples (corresponding to 60 seconds $\times 64$ channels), a learning rate of 0.002 , and 3000 iterations. To avoid over-fitting, the dropout technique from [18] was used with a ratio of 0.25 , which corresponds to randomly setting $25 \%$ of the hidden units to 0 . The network was implemented in Keras [19]. For testing, the correlation coefficients were obtained using the same correlation window setting as used for the least-squares-based AAD method.

For the state space model, the hyperparameters $c_{0}$ in 8 and $a_{0}$ and $b_{0}$ in (10) were set to $c_{0}=1, a_{0}=2.008$ and $b_{0}=0.2016$, similarly as in [7]. For testing, a sliding smoothing window of length $K_{\mathrm{SSM}}=3$ with $K_{A}=1$, $K_{P}=1$ was used. For each testing condition, the parameter set of the attended Log-Normal distribution $\boldsymbol{\alpha}_{a}$ in (11) was initialized by fitting over correlation coefficients of the (oracle) attended speaker obtained during the first $15 \mathrm{~s}$ and was then fixed. The parameter set of the unattended LogNormal distribution $\boldsymbol{\alpha}_{u}$ in (12) was similarly initialized by fitting over correlation coefficients of the (oracle) unattended speaker. For testing, the pa- 

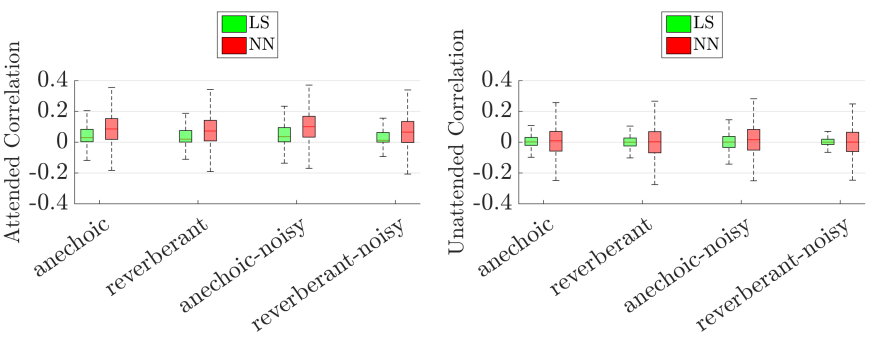

Fig. 2. Attended correlation and unattended correlation for different acoustic conditions when using the least-squares-based method and the neural-network-based AAD method.

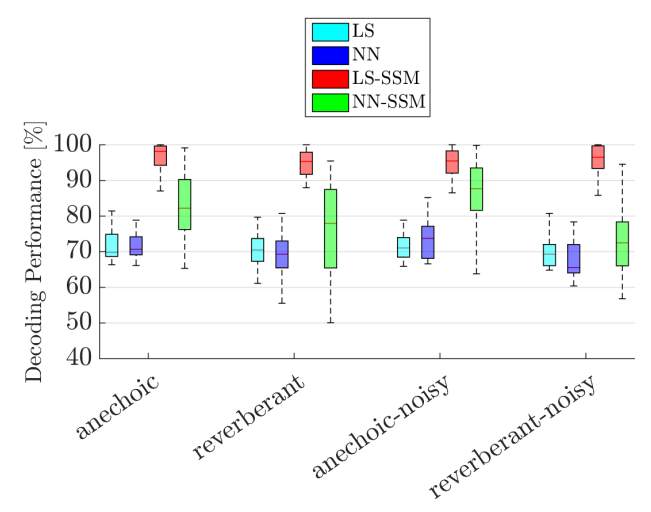

Fig. 3. Decoding performance for different acoustic conditions when using the least-squares-based method, the neural-network-based AAD method, the state-space model with the least-squares-based method and the state-space model with the neural-network-based AAD method.

rameters of the state-space model $\Omega$ corresponding to an smoothing window were estimated using the EM estimation algorithm with 20 iterations.

The quality of correlation coefficients generated by either the leastsquares-based AAD method or the neural-network-based AAD method was evaluated in terms of the attended correlation and the unattended correlation. The attended correlation was computed using the Pearson correlation between the reconstructed envelopes and the envelopes of the attended speaker, i.e., $\rho_{a, k}=\rho\left(\mathbf{e}_{a}[k], \hat{\mathbf{e}}_{a}[k]\right)$. The unattended correlation was computed between the reconstructed envelopes and the envelopes of the unattended speaker, i.e. $\rho_{u, k}=\rho\left(\mathbf{e}_{u}[k], \hat{\mathbf{e}}_{a}[k]\right)$,

The decoding performance was evaluated for several AAD methods, i.e., the least-squares-based AAD method (LS), the neural-network-based AAD method (NN), the state-space model using with the least-squaresbased AAD method (LS-SSM) and the state-space model with the neural-network-based AAD method (NN-SSM). The decoding performance for the least-squares-based and the neural-network-based AAD method was computed as the percentage of correctly decoded 5 -second correlation windows. The decoding performance for the state-space model using either the least-squares-based or the neural-network-based AAD method was computed as the percentage of correctly decoded 5 -second smoothing windows.

\section{RESULTS AND DISCUSSION}

For the least-squares-based AAD method and the neural-network-based AAD method, Fig. 2 depicts the attended correlation and the unattended correlation for different acoustic conditions. It can be observed for all acoustic conditions that the attended correlation obtained by the neuralnetwork-based AAD method (NN) is larger than the least-squares-based AAD method (LS), showing that the neural-network-based AAD method is able to reconstruct the attended speech envelope with a better accuracy. However, the attended correlation obtained by the neural-network-based $\mathrm{AAD}$ has a larger variability compared to the least-squares-based AAD method, which corresponds to attended correlation coefficients with a larger fluctuation. In addition, it can be observed that there is no significant difference in the unattended correlation obtained by the leastsquares-based AAD method and the neural-network-based AAD method. However, the unattended correlation obtained by the neural-networkbased AAD has a larger variability compared to the least-squares-based AAD method.

For all acoustic conditions, Fig. 3 depicts the decoding performance when using either the least-squares-based AAD method, the neural-network-based AAD method, the state-space model with the least-squares-based AAD method, or the state-space model with neuralnetwork-based AAD method. It can be observed that when using either the least-squares-based AAD method or the neural-network-based AAD method, a relatively low decoding performance (with the median decoding performance $69 \%-73 \%$ ) is obtained, mainly due to quite small (attended and unattended) correlations with a large variability (see Fig. 2) based on which decoding is performed by these methods. A statistical multiple comparison test (Kruskal-Wallis test followed by the posthoc Dunn and Sidak test [20]) revealed no significant difference $(p>0.05)$ in decoding performance when using the least-squares-based AAD method or the neural-network-based AAD method. When using the state-space model with either the least-squares-based or the neural-network-based AAD method (LS-SSM, LS-NN), the decoding performance increases. The increase is considerably larger for the least-squares-based AAD method (with the median decoding performance > 94\%) compared to the neural-network-based AAD method (with the median decoding performance $>73 \%$ ). The larger decoding performance can be explained by the fact that the correlations generated by the least-squares-based AAD method have a lower variability compared to the correlations generated by the neural-network-based AAD method, which leads to a smoother estimate of attention probabilities and a more stable decoding. The statistical multiple comparison test revealed that for most acoustic conditions (except anechoic-noisy) the decoding performance using the state-space model with the least-squares-based AAD method is significantly larger $(p<0.05)$ compared to using the least-squares-based AAD method, the neural-network-based AAD method, and the state-space model with the neural-network-based AAD method.

\section{CONCLUSION}

In this paper, we investigated a state-space model using correlation coefficients obtained with a 5-second correlation window to improve the decoding performance of the (linear) least-squares-based AAD method and the (non-linear) neural-network-based AAD method. The state-space model translates correlation coefficients, generated either by the leastsquares-based or the neural-network-based AAD method, into smooth estimates of the attention state. The experimental results showed for all acoustic conditions that there is no significant difference in decoding performance between using the least-squares-based AAD method and the neural-network-based AAD method. However, when using the state-space model with the least-squares-based AAD method, for most acoustic conditions the decoding performance significantly improves. 


\section{REFERENCES}

[1] J. O'Sullivan, Z. Chen, J. Herrero, G. M. McKhann, S. A. Sheth, A. D. Mehta, and N. Mesgarani, "Neural decoding of attentional selection in multi-speaker environments without access to clean sources," Journal of Neural Engineering, vol. 14, no. 5, p. 56001, 2017.

[2] S. Van Eyndhoven, T. Francart, and A. Bertrand, "EEG-informed attended speaker extraction from recorded speech mixtures with application in neuro-steered hearing prostheses," IEEE Transactions on Biomedical Engineering, vol. 64, no. 5, pp. 1045-1056, 2017.

[3] A. Aroudi and S. Doclo, "Cognitive-driven binaural beamforming using EEG-based auditory attention decoding," IEEE Transactions on Audio, Speech, and Language Processing, in press.

[4] J. A. O'Sullivan, A. J. Power, N. Mesgarani, S. Rajaram, J. J. Foxe, B. G. Shinn-Cunningham, M. Slaney, S. A. Shamma, and E. C. Lalor, "Attentional selection in a cocktail party environment can be decoded from single-trial EEG," Cerebral Cortex, 2014.

[5] D. D. Wong, S. A. Fuglsang, J. Hjortkjær, E. Ceolini, M. Slaney, and A. de Cheveigné, "A comparison of regularization methods in forward and backward models for auditory attention decoding," Frontiers in Neuroscience, vol. 12, p. 531, 2018. [Online]. Available: https://www.frontiersin.org/article/10.3389/fnins.2018.00531

[6] E. Alickovic, T. Lunner, F. Gustafsson, and L. Ljung, "A tutorial on auditory attention identification methods," Frontiers in Neuroscience, vol. 13, p. 153, 2019.

[7] S. Miran, S. Akram, A. Sheikhattar, J. Z. Simon, T. Zhang, and B. Babadi, "Real-time tracking of selective auditory attention from M/EEG: A Bayesian filtering approach," Frontiers in Neuroscience, vol. 12, p. 262, 2018.

[8] T. de Taillez, B. Kollmeier, and B. T. Meyer, "Machine learning for decoding listeners attention from electroencephalography evoked by continuous speech," European Journal of Neuroscience, Dec. 2018.

[9] G. Ciccarelli, M. Nolan, J. Perricone, P. T. Calamia, S. Haro, J. OSullivan, N. Mesgarani, T. F. Quatieri, and C. J. Smalt, "Comparison of two-talker attention decoding from EEG with nonlinear neural networks and linear methods," Scientific Reports, Nature, vol. 9, no. 11538, Aug. 2019.

[10] A. Aroudi and S. Doclo, "Cognitive-driven binaural LCMV beamformer using EEG-based auditory attention decoding," in Proc. IEEE International Conference on Acoustics, Speech and Signal Processing (ICASSP), Brighton, United Kingdom, May 2019, pp. 406-410.

[11] C. Han, J. O'Sullivan, Y. Luo, J. Herrero, A. D. Mehta, and N. Mesgarani, "Speaker-independent auditory attention decoding without access to clean speech sources," Science Advances, vol. 5, no. $5,2019$.

[12] S. Akram, J. Z. Simon, and B. Babadi, "Dynamic estimation of the auditory temporal response function from MEG in competingspeaker environments," IEEE Transactions on Biomedical Engineering, vol. 64, no. 8, pp. 1896-1905, 2017.

[13] A. Aroudi and S. Doclo, "EEG-based auditory attention decoding using unprocessed binaural signals in reverberant and noisy conditions," in Proc. Int. Conf. of the IEEE Engineering in Medicine and Biology Society (EMBC), Jeju, South Korea, 2017, pp. 484-488.

[14] H. Kayser, S. D. Ewert, J. Anemüller, T. Rohdenburg, V. Hohmann, and B. Kollmeier, "Database of multichannel in-ear and behind-theear head-related and binaural room impulse responses," EURASIP Journal on Advances in Signal Processing, vol. 2009, p. 6, 2009.
[15] E. Habets, I. Cohen, and S. Gannot, "Generating nonstationary multisensor signals under a spatial coherence constraint," Journal of the Acoustical Society of America, vol. 124, no. 5, pp. 2911-2917, Nov. 2008.

[16] A. Aroudi, B. Mirkovic, M. De Vos, and S. Doclo, "Impact of different acoustic components on EEG-based auditory attention decoding in noisy and reverberant conditions," IEEE Transactions on Neural Systems and Rehabilitation Engineering, vol. 27, no. 4, pp. 652-663, April 2019.

[17] T. Dozat, "Incorporating nesterov momentum into adam," in International Conference on Learning Representations (ICLR 2016 workshop), 2016.

[18] N. Srivastava, G. Hinton, A. Krizhevsky, I. Sutskever, and R. Salakhutdinov, "Dropout: A simple way to prevent neural networks from overfitting," Journal of Machine Learning Research, vol. 15, pp. 1929-1958, 2014.

[19] F. Chollet et al., "Keras," https://keras.io 2015.

[20] Y. Hochberg and A. C. Tamhane, Multiple Comparison Procedures. John Wiley and Sons, 1987. 